\title{
From crystalline to exotic arrangements of matter in neutron star crusts
}

\author{
V. De la Mota, F. Sébille, S. Figerou and Ph. Eudes
}

\begin{abstract}
SUBATECH, Ecole des Mines, Université de Nantes, CNRS/IN2P3, Nantes, France
\end{abstract}

\begin{abstract}
We investigate the occurrence of exotic structures in the outermost layers of neutron stars within the framework of a microscopic model describing the nucleonic dynamics through a time-dependent mean field approach at around zero temperature. In this model starting from an initial crystalline lattice of nuclei at subnuclear densities the system evolves and self-organizes in various low-lying energy structures without assumption of final shapes. These structures are studied in terms of a density phase diagram. We investigate their sensitivity to the isotopic composition and to the symmetries of the lattice.
\end{abstract}

\section{Introduction}

Neutron stars are cold, dense and compact objects supported by the degeneracy pressure of neutrons. At its outermost layers, temperatures are $\mathrm{T} \ll 1$ $\mathrm{MeV}$ while densities are around $\rho \sim 10^{11} \mathrm{~g} \mathrm{~cm}^{-3}$. As predicted in the early 80 's, in these regions nuclei may adopt exotic shapes. They are assumed to be built as the consequence of the interplay between Coulomb and nuclear forces and to be sensitive to the equation of state (EOS) of nuclear matter. Their existence may have important astrophysical consequences on macroscopic characteristics, as masses and radii [1], as well as on the cooling processes of the star [2]. In particular, their presence should affect the neutrino opacity [3] and the transport properties [4] of the crust. 
In order to describe the behavior of the crustal matter we have developed a dynamical model accounting for nucleonic degrees of freedom [5]. In this framework, non spherical shapes are shown to appear at subnuclear densities as a consequence of the nuclear micro-dynamics. They correspond to multiple low-lying energy configurations that can be explored dynamically starting from different initial conditions. As originally predicted by static approaches [6], nuclei with exotic shapes as rods, slabs and other complex structures are observed.

The aim of this work is to investigate the self-organization processes of the nuclear matter from the lattice structures expected in the outer crust to either exotic structures or disordered patterns in the inner crust. The temporal evolution of the nucleon density is analyzed for different isotopic compositions, mean densities and symmetries. The effects of random perturbations in nuclei positions are also examined. This work is organized as follows. In Section 2 we briefly recall the bases of the model. In Section 3 we discuss our results. In Section 3 we present our conclusions and perspectives.

\section{The model}

In the range of temperatures and densities characteristics of the star, matter is described in terms of a system of interacting nucleons in a uniform background of electrons insuring the neutrality of matter. We have developped a dynamical approach which is based on the DYWAN model [7] describing heavy ion collisions.

In the present approach, the initial condition is given by a system of nuclei located on the sites of a lattice with periodic boundary conditions. A static self-consistent procedure is implemented in order to get nuclear composites either at their ground states or in excited states according to mechanical or thermal constraints. The Hartree-Fock equation is solved spanning the one-body density matrix in a convenient basis (wavelets [8]). For this calculation we have chosen a density-dependent zero-range effective interaction, with the following self-consistent field:

$$
\begin{aligned}
V_{q}^{H F}(\rho, \xi)= & \frac{t_{0}}{\rho_{\infty}} \rho+\frac{t_{3}}{\rho_{\infty}^{7 / 6}} \rho^{7 / 6}+\frac{c}{\rho_{\infty}^{2}} \xi^{2}+\frac{4 q c}{\rho_{\infty}^{2}} \rho \xi+ \\
& \frac{\Omega}{3 \rho_{\infty}^{2}} \xi^{2}+\frac{4 q \Omega}{3 \rho_{\infty}^{2}}\left(\rho-\rho_{\infty}\right) \xi+V_{q}^{C}
\end{aligned}
$$

where $\rho_{n}$ and $\rho_{p}$ stand for neutron and proton densities, $\rho=\rho_{n}+\rho_{p}$, $\xi=\rho_{n}-\rho_{p}, q=1 / 2$ for neutrons and $-1 / 2$ for protons, $\rho_{\infty}=0.145 \mathrm{fm}^{-3}$ 
is the saturation density of infinite nuclear matter and $V_{q}^{C}$ is the Coulomb interaction. Current values of the parameters are $c=20 \mathrm{MeV}, \Omega=-100 \mathrm{MeV}$, $t_{0} / \rho_{\infty}=-356 \mathrm{MeV} \mathrm{fm}^{3}$ and $t_{3} / \rho_{\infty}^{7 / 6}=303 \mathrm{MeV} \mathrm{fm}^{7 / 2}$. They are shown to reproduce the principal static characteristics of nuclei and of infinite neutron matter [5]. The Coulomb term for protons is calculated using Ewald summation techniques [9], which are adapted to the calculation of long range potentials in periodic systems.

Once the initial conditions are given nuclear matter evolves according to the Time Dependent Hartree-Fock equation. A variational procedure is implemented in order to obtain the equations of motion for the basis parameters $\{\vec{\xi}(t), \vec{\chi}(t), \vec{\pi}(t), \vec{\phi}(t)\}$ corresponding to the first and second moments in phase space: $\langle x\rangle=\xi_{x},\left\langle\left(x-\xi_{x}\right)^{2}\right\rangle=\chi_{x},\left\langle p_{x}\right\rangle=\pi_{x}$, $<\left(p_{x}-\pi_{x}\right)^{2}>=\phi_{x}$. We refer the reader to Ref. [5] for a detailed description of the model.

The nucleon distribution evolves in time molding structures which can strongly differ from the initial ordered condition. Morphological analysis techniques (MIA) [10] provides a mathematical framework to classify structures unambiguously. The associated Euler characteristics is defined as $\chi=n_{e}-n_{t}+n_{c}$, where $n_{e}$ is the number of elements, $n_{t}$ is the number of tunnels and $n_{c}$ is the number of cavities. Table 1 summarizes the obtained basic shapes with their corresponding $\chi$ value.

Table 1: Synopsis of basic Euler characteristic values.

\begin{tabular}{|c|c|c|c|c|c|c|c|}
\hline$\chi_{E}$ & 27 & 9 & 3 & -3 & -9 & -15 & 27 \\
\hline Structures & spheres & $\begin{array}{c}\text { rods and } \\
\text { spherical holes }\end{array}$ & slabs & $\begin{array}{c}\text { cylindrical } \\
\text { bubbles }\end{array}$ & $\begin{array}{c}\text { slabs } \\
\text { with holes }\end{array}$ & $\begin{array}{c}\text { connected } \\
\text { slabs }\end{array}$ & sponges \\
\hline
\end{tabular}

\section{Results}

In Fig. 1 are displayed a set of chosen snapshots of the neutron density contained in a supercell, at different times, for the simple cubic cell (SCC) lattice containing 27 oxygen nuclei with proton fraction $x_{p}=0.1$. Nuclei have been initially slightly excited with a quadrupole deformation. The overall average density of the supercell is $\langle\rho\rangle=0.0726 \mathrm{fm}^{-3}$. In this picture only spatial regions with densities higher than the threshold density $\rho_{t}=0.065$ $\mathrm{fm}^{-3}$ are apparent. In Fig. 2 (a) the corresponding $\chi$ values are plotted as 

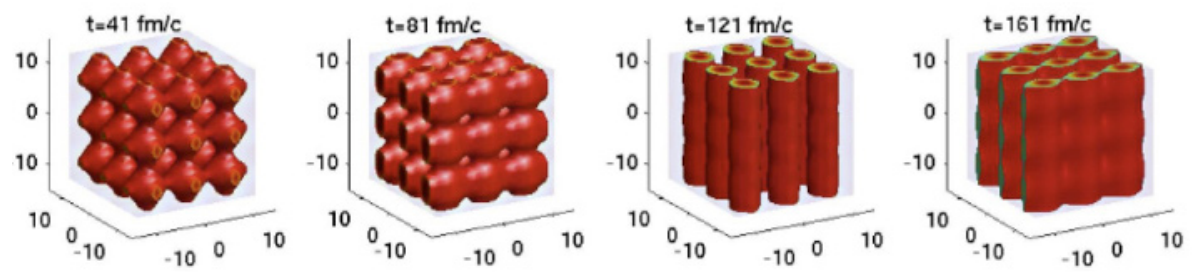

Figure 1: (Colour on line) Time evolution of neutron density in oxygen SCC lattice with $x_{p}=0.1,\langle\rho\rangle=0.0726 \mathrm{fm}^{-3}$ and threshold density $0.065 \mathrm{fm}^{-3}$.

a function of time. After a transient interval the system oscillates between two preferred structures. These transitions are energy and entropy conserving. In Fig. 2 (b) is represented the entropy per baryon in units of the Boltzmann constant as a function of time. When, in addition, the system is
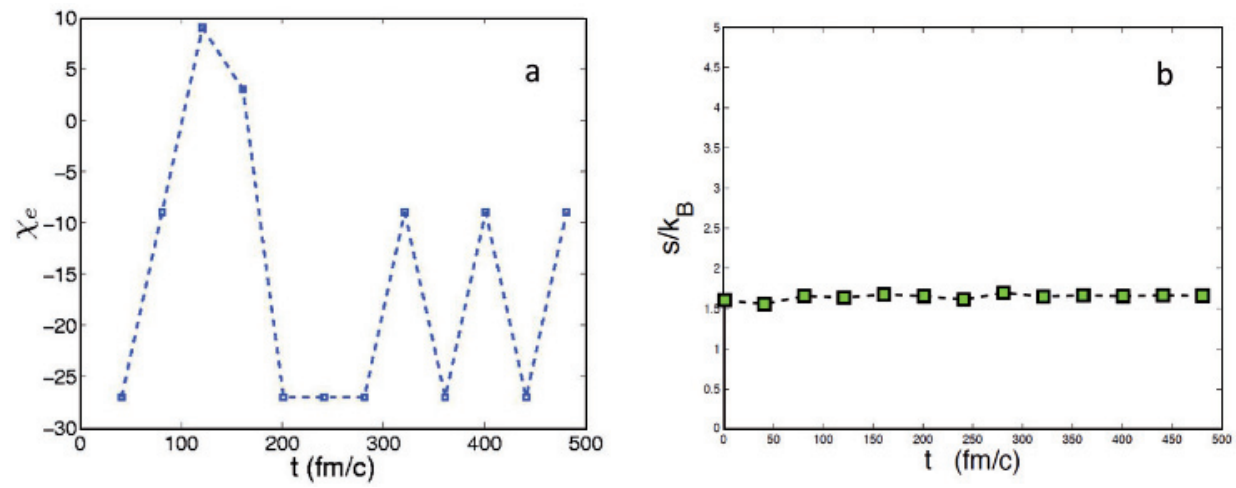

Figure 2: (Colour on line) Same system as in Fig. 1, the time evolution of (a) the Euler characteristics and (b) the entropy per nucleon. $\mathrm{K}_{B}$ is the Boltzmann constant.

initially perturbed by slightly shifting the positions of nuclei with respect to the lattice sites, the subsequent evolution strongly depends on the isotopic composition and on the spatial symmetry of the system. Indeed, in Fig. 3 is represented the neutron density distribution in a perturbed SCC supercell of $\mathrm{O}$ isotopes with proton fractions $x_{p}=0.5$ (a) and $x_{p}=0.2(\mathrm{~b})$. The mean and threshold densities are, respectively, $0.06 \mathrm{fm}^{-3}$ and $0.04 \mathrm{fm}^{-3}$. Starting from similar initial conditions the system evolves toward different configurations. In the case (a) the system organizes in several intermediate mass fragments while for the case (b) it turns into a unique massive cluster occupying the entire cell. If, instead of using SCC lattices, face centered cubic 

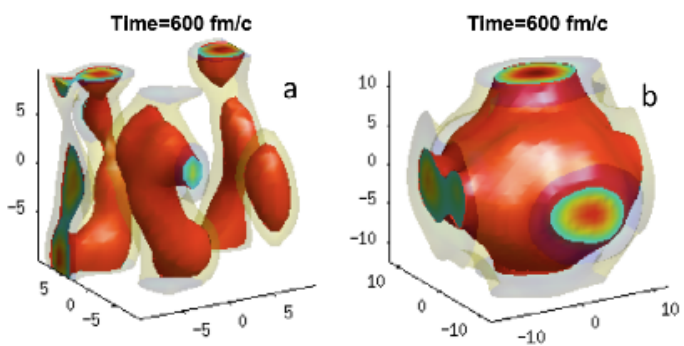

Figure 3: (Colour online) Snapshots of neutron density at $\mathrm{t}=600 \mathrm{fm} / \mathrm{c}$ in perturbed oxygen SCC lattice with $\langle\rho\rangle=0.06 \mathrm{fm}^{-3}$ for (a) $x_{p}=0.5$ and (b) $x_{p}=0.2$.

cells (FCC) are implemented, the system evolves toward a unique rod-like fragment. This is shown in Fig. 4, where is displayed the time evolution of the neutron density in a perturbed supercell composed by $32 \mathrm{O}$ nuclei with proton fraction $x_{p}=0.5$, the mean density being $\langle\rho\rangle=0.06 \mathrm{fm}^{-3}$ and $\rho_{t}=0.04 \mathrm{fm}^{-3}$. Despite of the random perturbations imposed to nuclei po-
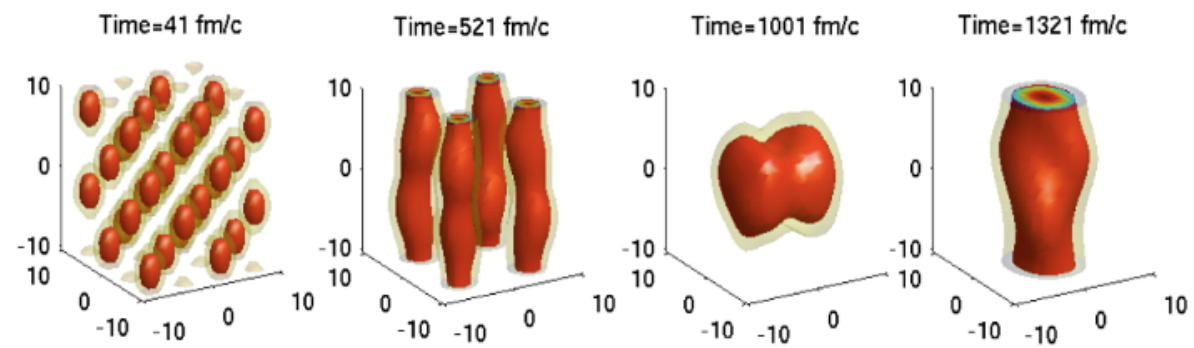

Figure 4: (Colour online) Time evolution of neutron density in oxygen FCC lattice with $x_{p}=0.5,\langle\rho\rangle=0.06 \mathrm{fm}^{-3}$ and threshold density $0.04 \mathrm{fm}^{-3}$.

sitions, the system attempts to preserve symmetries and develops stringy structures. At times $\mathrm{t} \sim 1300 \mathrm{fm} / \mathrm{c}$, the dominant arrangements are infinite rod-like structures.

Phase diagrams in the $\left(\rho_{t},\langle\rho\rangle\right)$ plane give a concise view of the occurrence of different structures and of their relative weight. The phase diagram corresponding to the above oxygen lattice of Fig. 4 has been plotted in Fig. 5. The presence of cylindrical structures is shown to be dominant for average densities higher than about $0.3 \rho_{\infty}$. Lowering the threshold density, and for narrow bins of it, other structures like slabs and bubble-like are also present. The non uniformity of the density distribution is also evidenced at higher density thresholds where spherical-like structures are shown to be 


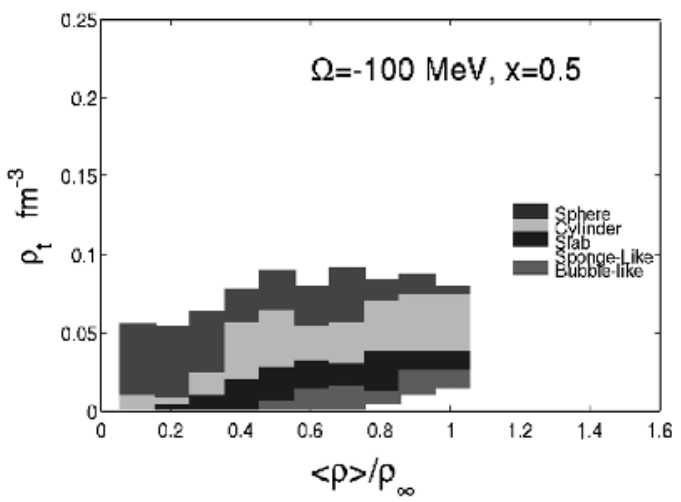

Figure 5: Structure diagram corresponding to the system in Fig. 4.

present. Generally speaking, the occurrence of heavy aggregates is deeply related with the ability of the model to describe the spreading of wave functions widths corresponding to unbounded nucleons. This trend is enhanced at low proton fractions.

\section{Conclusions}

In this work we presented a model describing the dynamics of nuclear matter in the crust of a neutron star. In this approach the dynamical processes in inhomogeneous nuclear matter can be simulated using a large number of nucleons without shape assumptions, in contrast to the many previous studies employing static frameworks. Starting from different initial lattices of oxygen nuclei with different proton fractions, mean densities and cell geometries, not only the standard types of pasta emerge naturally but also a large variety of energetically equivalent intermediate shapes. The slight initial excitation allows the system to explore the landscape of structures. Since the evolution is reversible, the the total entropy per baryon remains constant and lattice symmetries are preserved in time. The effects of perturbing at random nuclei positions is to destroy symmetries in the case with $x_{p}=0.5$. Here a variety of irregularly distributed intermediate mass clusters are found. In the case of the low proton fraction $x_{p}=0.2$ the system aggregates in a single heavy cluster holding the overall supercell. We have studied the evolution of perturbed FCC oxygen lattices with proton fraction 0.5. The corresponding density evolution for one particular choice of $\langle\rho\rangle$ and the phase diagram in the $\rho_{t}$ versus $\langle\rho\rangle$ plane reveal the occurrence of cylindrical structures besides quasi-spherical nuclei at sufficiently long 
times. The occurrence of heavy aggregates, notably at low proton fraction, is related with the capacity of the model to describe the spreading of wave functions widths corresponding to unbounded nucleons.

The formation and transitions between non-equilibrium structures may strongly depend on the nature of nucleon interactions. Investigations of the structure landscape in inhomogeneous nuclear matter at subnuclear densities using more complex non-local effective forces and beyond a pure mean-field description are currently in progress.

\section{References}

[1] G. Haensel, A.Y. Potekhin and D.G. Yakovlev; Neutron Stars 1, Springer (2007).

[2] D. Page, U. Geppert, F. Weber, Nucl. Phys. A 777 (2006) 497.

[3] C.J. Horowitz, M.A. Perez-Garcia, J. Piekarewicz, Phys. Rev. C 69 (2004) 045804.

[4] H. F. Zhang, U. Lombardo and W. Zuo, Phys. Rev. C 82 (2010) 015805.

[5] F. Sébille, S. Figerou and V. de la Mota, Phys. Nucl. A 822 (2009) 51; F. Sébille, V. de la Mota and S. Figerou, Phys. Rev. C 84 (2011) 055801.

[6] D.G. Ravenhall, C.J. Pethick and J.R. Wilson, Phys. Rev. Lett. 50 (1983) 2066; M. Hashimoto, H. Seki and M. Yamada, Prog. Theor. Phys. 71 (1984) 320.

[7] B. Jouault, F. Sébille and V. de la Mota, Nucl. Phys. A 628 (1998) 119.

[8] I. Daubechies, Ten lectures on wavelets, Ams Providence (1992).

[9] A. Y. Toukmaji and J.A. Board Jr., Comp. Phys. Comm. 95 (1996) 73.

[10] K. Michielsen and H. De Raedt, Phys. Rep. 347 